\title{
Elevated POLR2F expression distinguishes basal-like human breast cancer.
}

Shahan Mamoor, MS ${ }^{1}$

$3 \quad$ shahanmamoor@gmail.com

East Islip, NY 11730

Patients diagnosed with basal-like breast cancer face a more aggressive disease course and more dismal prognosis than patients diagnosed with luminal A and luminal B breast cancer molecular subtypes (1-4). We mined published microarray data $(5,6)$ to understand in an unbiased fashion the most distinguishing transcriptional features of tumors from patients with basal or basal-like subtype breast cancer. We observed transcriptome-wide differential expression of RNA polymerase II subunit F, POLR2F, when comparing tumors of patients with basal-like breast cancer with that of other PAM50 molecular subtypes. POLR2F mRNA was present at significantly higher quantities in the tumors of patients with basal-like breast cancer. Analysis of patient survival data revealed that POLR2F primary tumor expression was correlated with recurrence-free survival in basal subtype breast cancer. Elevated POLR2F expression appears to distinguish basal-like human breast cancer from the other molecular subtypes.

Keywords: basal subtype, basal-like breast cancer, POLR2F, systems biology of breast cancer, targeted therapeutics in breast cancer, molecular subtype 
Classification of disease types and disease subtypes enables development of enhanced diagnostic capabilities, acquisition of prognostic information, as well as an understanding of human disease that can support superior medical, surgical and radiological treatment of complex diseases such as cancer. Molecular subtypes in human breast cancer include luminal A, luminal B, basal or basal-like, and HER2+. Systems-level analyses, such as comparative tumor transcriptome analyses, can inform such approaches. We mined published microarray data $(5,6)$ to identify the most significant transcriptional differences when comparing human breast cancer molecular subtypes. Integrative microarray dataset analysis has provided data illuminating genes whose expression define basal-like subtype breast cancers, and we present evidence here.

\section{Methods}

We utilized datasets GSE74667 (5) and GSE87049 (6) for this global differential gene expression analysis of basal subtype human breast cancer in conjunction with GEO2R. GSE74667 was generated using Agilent-014850 Whole Human Genome Microarray 4x44K G4112F technology with $n=23$ basal-like breast tumors and $n=72$ breast tumors of other molecular subtypes (luminal A, luminal B, HER2-enriched, and normal-like); analysis was performed using platform GPL6480. GSE87049 was generated using Affymetrix Human Gene 1.0 ST Array [transcript (gene) version] technology with $n=14$ basal-like breast tumors and $n=109$ breast tumors of other molecular subtypes (luminal A, luminal B, HER2-enriched, and normal-like); analysis was performed using platform GPL6244. The Benjamini and Hochberg method of $p$-value adjustment was used for ranking of differential expression but raw $p$-values were used to assess statistical significance of global differential expression. Log-transformation of data was auto-detected, and the NCBI generated category of platform annotation was used. A statistical test was performed to evaluate whether POLR2F gene expression was significantly different between the tumors of patients with basal and non-basal molecular subtype breast cancer using a two-tailed t-test with Welch's correction (PRISM 9.1.1 (225)). For Kaplan-Meier survival analysis, we used the Kaplan-Meier plotter tool (8) for correlation of POLR2F mRNA expression levels with recurrence-free survival in $n=4929$ breast cancer patients, $n=953$ basal subtype breast cancer patients, $n=1809$ luminal A subtype breast cancer patients, $n=1353$ luminal B subtype breast cancer patients, $n=695$ HER $2+$ subtype breast cancer patients, and $n=119$ normal-like subtype breast cancer patients.

\section{Results}

POLR2F is among the genes whose expression is most different when comparing basal-like breast cancers with breast cancers of other molecular subtypes.

We used published microarray data (5) to compare the whole tumor transcriptomes of 23 patients with basal subtype breast cancer and 72 patients with breast cancer of other molecular subtypes (luminal A, luminal B, HER2+ and normal-like) to identify genes whose expression marked the basal-like molecular subtype. We observed transcriptome-wide differential expression of RNA polymerase II subunit F, POLR2F, when comparing the tumors of patients with basal-like cancer with that of patients with luminal A, luminal B and HER2+ cancers (Table 1). When sorting each of the transcripts whose expression was measured globally, POLR2F expression ranked 188 out of 31157 total transcripts, equating to $99.4 \%$ differential expression; this was statistically significant ( $p$-value: $2.47 \mathrm{E}-09$ ).

Analysis of a second published microarray dataset, comparing tumor transcriptome data from a separate cohort of patients (14 patients with basal-like breast cancer and 109 patients with breast cancer of other molecular subtype (luminal A, luminal B, HER2+ and normal-like) (5) revealed transcriptome-wide differential expression of POLR2F in patients with basal subtype human breast cancer (Table 2). When sorting each of the transcripts whose expression was measured globally, POLR2F expression ranked 172 
out of 33297 total transcripts, equating to $99.5 \%$ differential expression; this was statistically significant ( $p$-value: 6.65E-13). Thus, the data suggested differential expression of POLR2F was a fundamental feature of basal-like breast cancer in humans rather than an artifact of a single microarray dataset or research group, or a finding derived from cell culture or an animal model that was not relevant to human breast cancer.

POLR2F expression is significantly higher in basal-like breast cancers.

We obtained exact POLR2F mRNA expression data for basal-like breast tumors and tumors from other molecular subtypes (luminal A, luminal B, HER2+ and normal-like) to determine magnitude and direction of POLR2F gene expression change in basal-like breast cancer. POLR2F was expressed at higher levels in the tumors of patients with basal-like breast cancer as compared to the tumors of patients from other PAM50 molecular subtypes, and this difference was statistically significant (Figure 1; $p<0.0001$ ). POLR2F was expressed at $0.233 \pm 0.493$ arbitrary units (A.U.) in basal-like breast cancer and $-0.388 \pm 0.320$ A.U. in all other molecular subtypes (Figure 1).

POLR2F expression correlates with recurrence-free survival in basal-like breast cancer.

We asked whether primary tumor expression of POLR2F was correlated with survival outcomes in patients with breast cancer when analyzing outcomes based on PAM50 molecular subtype. We observed a correlation between primary tumor expression of POLR2F and recurrence-free survival (RFS) in patients with basal subtype breast cancer which was statistically significant (Figure 2; $p$-value: 0.03; hazard ratio: 1.27 (1.02-1.57)). POLR2F primary tumor expression was a negative prognostic indicator in patients with basal subtype breast cancer. Basal subtype patients with low tumor expression of POLR2F possessed median OS of 30.42 months, while basal subtype patients with high tumor expression of POLR2F possessed median OS of 18.35 months (Table 3).

We also observed a correlation between primary tumor expression of POLR2F and recurrence-free survival (RFS) in all patients with breast cancer, irrespective of molecular subtype, which was statistically significant (Figure 2; $p$-value: 3.5e-05; hazard ratio: 1.24 (1.12-1.37)). POLR2F primary tumor expression was a negative prognostic indicator in patients with breast cancer. Patients with low tumor expression of POLR2F possessed median OS of 59 months, while patients with high tumor expression of POLR2F possessed median OS of 41.04 months (Table 3 ).

POLR2F primary tumor expression was not correlated with recurrence-free survival in luminal A subtype subtype breast cancer (Figure 2; $p$-value: 0.79 ; hazard ratio: 1.03 (0.84-1.26)), in luminal B subtype breast cancer (Figure 2; $p$-value: 0.62; hazard ratio: 1.05 (0.88-1.24), in HER2+ breast cancer (Figure 2; $p$-value: 0.13; hazard ratio: 1.2 (0.95-1.53)), or in normal-like breast cancer (Figure 2; $p$-value: 0.26 ; hazard ratio: $1.48(0.75-2.93))$.

\section{Discussion}

We find here that POLR2F is among the genes whose expression is most different when comparing basal-like breast cancers (BLBC) to cancers of other molecular subtypes, with significantly more POLR2F mRNA in BLBC. Kaplan-Meier analysis of patient survival data revealed that POLR2F expression was correlated with recurrence-free survival in patients with basal subtype breast cancer. The molecular function of POLR2F and any specific transcriptional tasks it participates in may be pertinent to tumor subtype identity in basal-like breast cancer. 


\section{References}

1. Rakha, E.A., Reis-Filho, J.S. and Ellis, I.O., 2008. Basal-like breast cancer: a critical review. Journal of clinical oncology, 26(15), pp.2568-2581.

2. Milioli, H.H., Tishchenko, I., Riveros, C., Berretta, R. and Moscato, P., 2017. Basal-like breast cancer: molecular profiles, clinical features and survival outcomes. BMC medical genomics, 10(1), pp.1-17.

3. Foulkes, W.D., Smith, I.E. and Reis-Filho, J.S., 2010. Triple-negative breast cancer. New England journal of medicine, 363(20), pp.1938-1948.

4. Dietze, E.C., Sistrunk, C., Miranda-Carboni, G., O'regan, R. and Seewaldt, V.L., 2015. Triple-negative breast cancer in African-American women: disparities versus biology. Nature Reviews Cancer, 15(4), pp.248-254.

5. She, Q.B., Gruvberger-Saal, S.K., Maurer, M., Chen, Y., Jumppanen, M., Su, T., Dendy, M., Lau, Y.K.I., Memeo, L., Horlings, H.M. and van de Vijver, M.J., 2016. Integrated molecular pathway analysis informs a synergistic combination therapy targeting PTEN/PI3K and EGFR pathways for basal-like breast cancer. BMC cancer, 16(1), pp.1-16.

6. Romero-Cordoba, S.L., Salido-Guadarrama, I., Rebollar-Vega, R., Bautista-Piña, V., Dominguez-Reyes, C., Tenorio-Torres, A., Villegas-Carlos, F., Fernández López, J.C., Uribe-Figueroa, L., Alfaro-Ruiz, L. and Hidalgo-Miranda, A., 2021. Comprehensive omic characterization of breast cancer in Mexican-Hispanic women. Nature communications, 12(1), pp.1-19.

7. Györffy, B., Lanczky, A., Eklund, A.C., Denkert, C., Budczies, J., Li, Q. and Szallasi, Z., 2010. An online survival analysis tool to rapidly assess the effect of 22,277 genes on breast cancer prognosis using microarray data of 1,809 patients. Breast cancer research and treatment, 123(3), pp.725-731. 


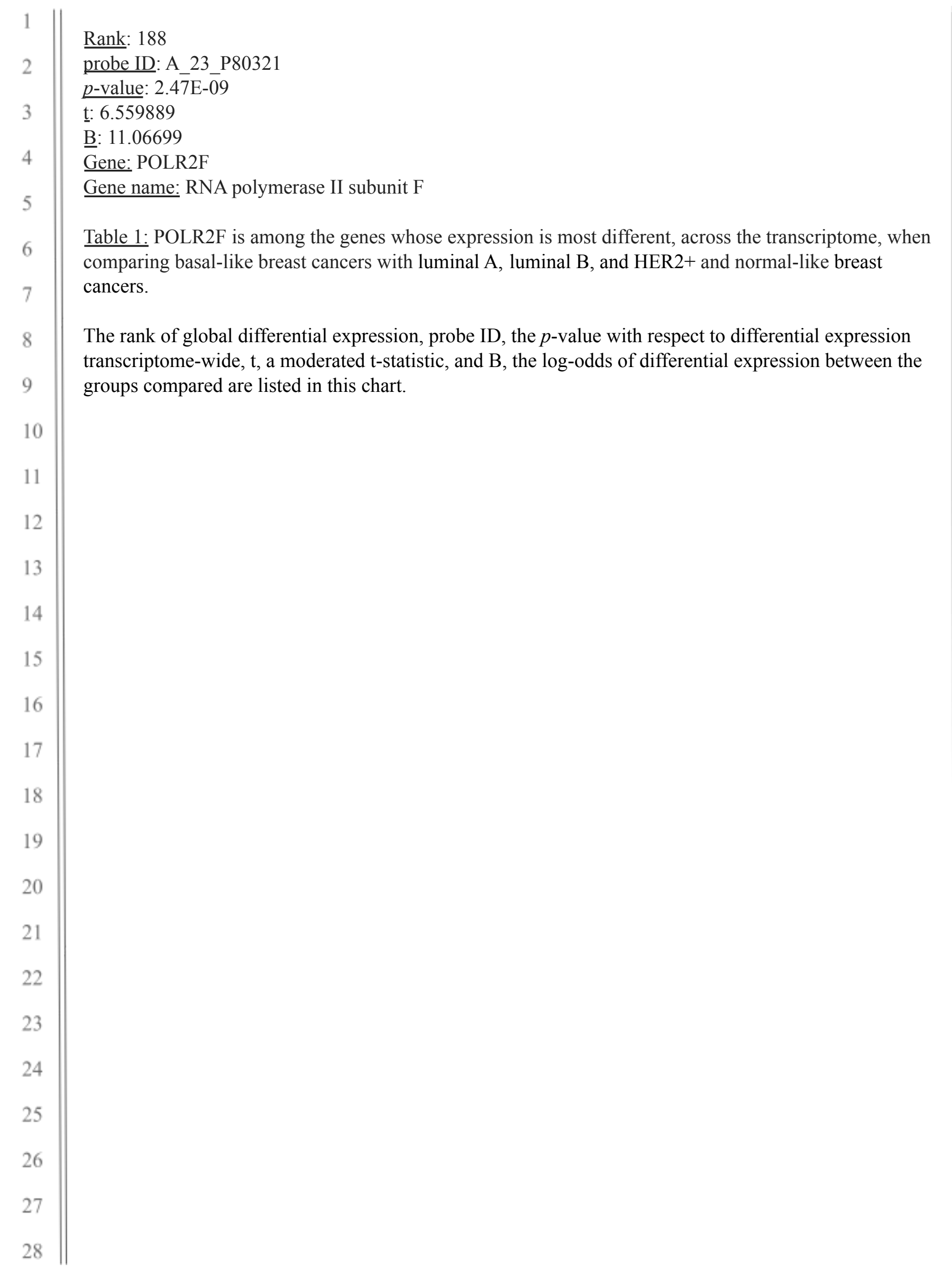




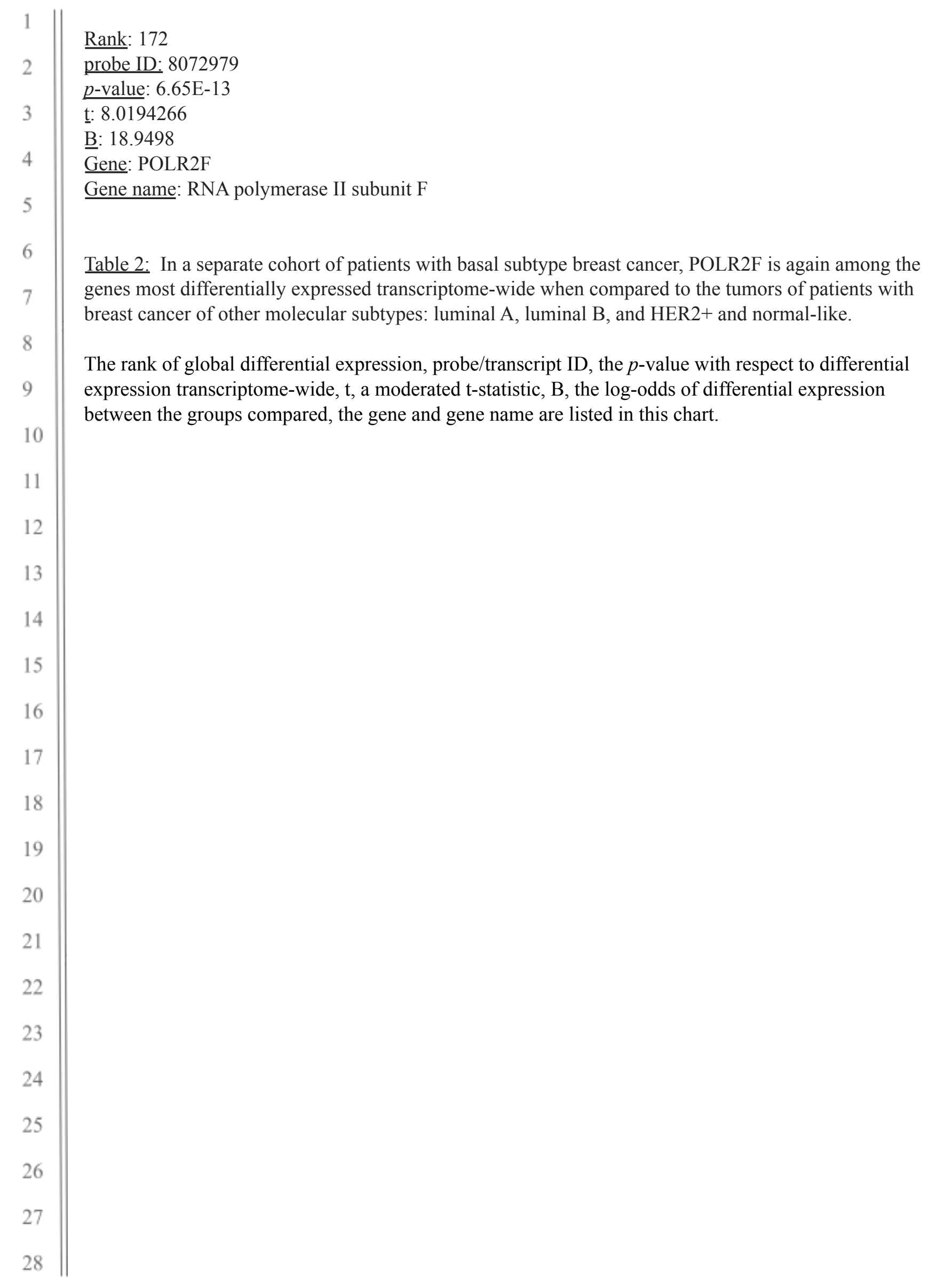




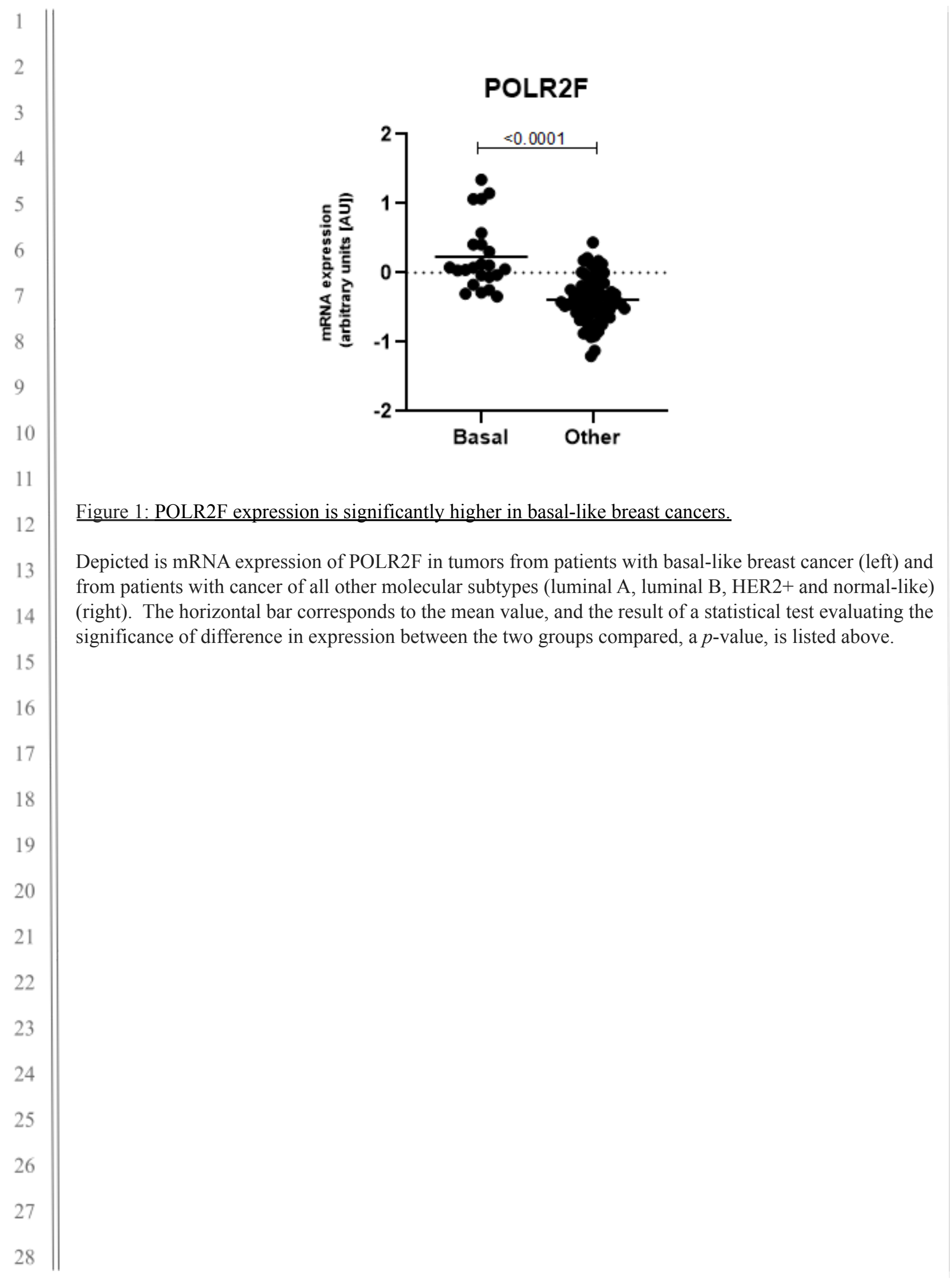




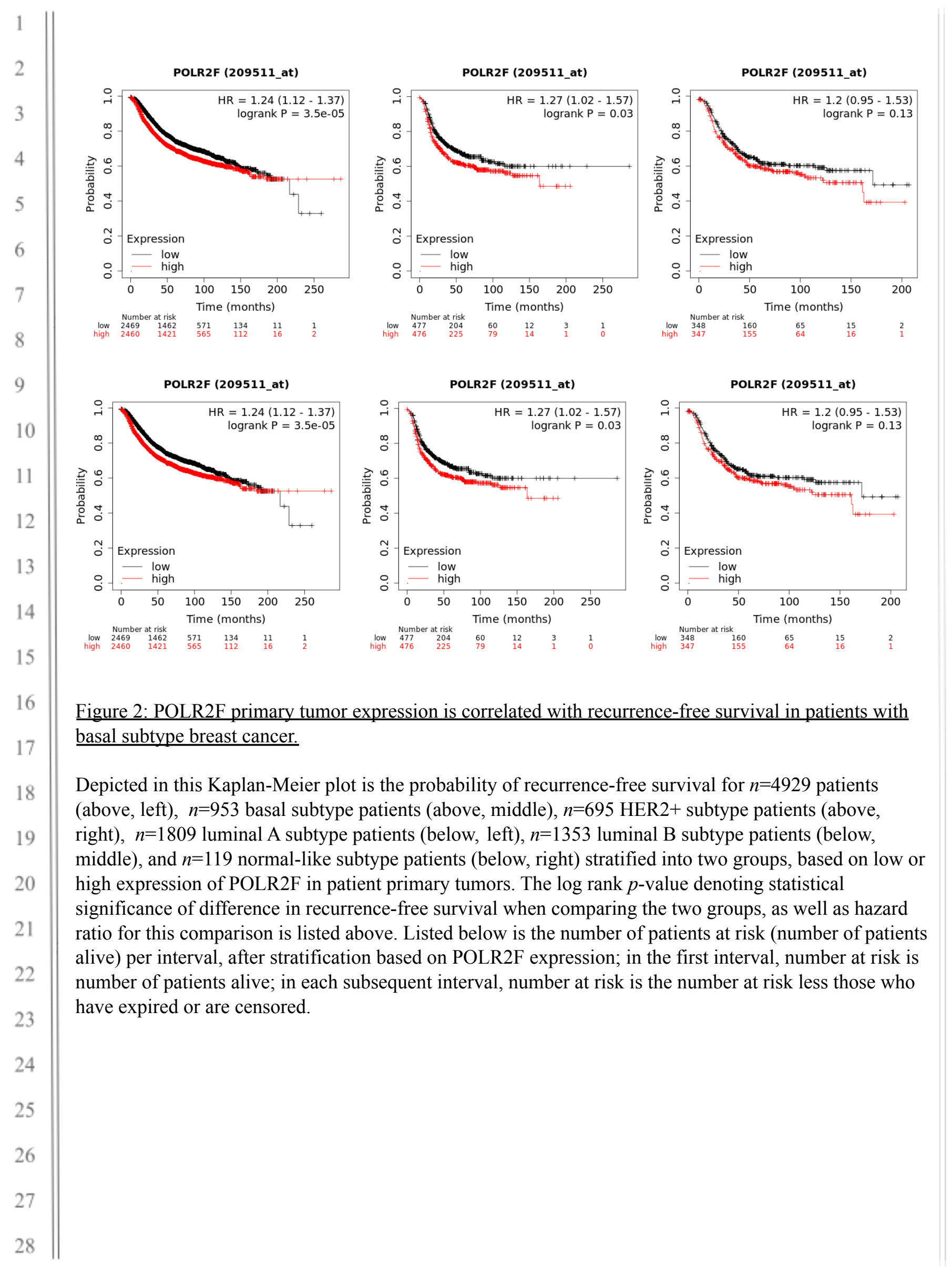




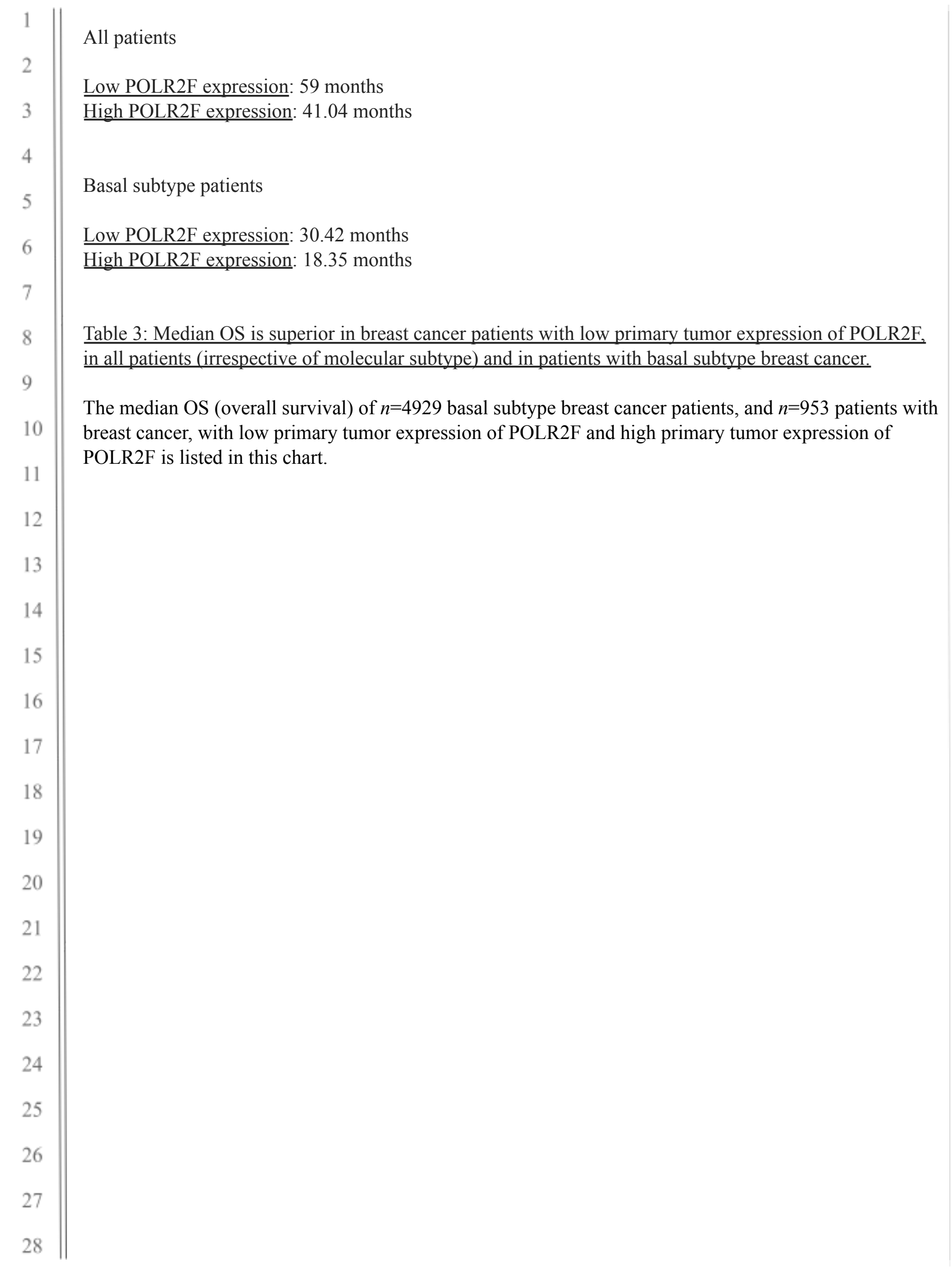

